\title{
Almost immersions
}

\author{
By Kazuaki Kobayashi
}

(Received February 12, 1975)

1. We work in the category of compact $P L$ spaces and $P L$ maps [Z]. Given spaces $X$ and $Y$ and a map $f: X \rightarrow Y$, we define $U_{r}(f)$ to be the set of points $x \in X$ such that $f^{-1} f(x)$ contains at least $r$ points. $S_{r}(f)$ is the closure of $U_{r}(f)$ in $X$. We call $B(f)=S_{2}(f)-U_{2}(f)$ branch locus of $f$ (i. e. for $x \in B(f) f^{-1} f(x)=x$ but $f \mid U(x)$ is not embedding for any neighborhood $U(x)$ of $x$.) Conversely we consider whether $f \mid U_{2}(f)$ is a local embedding (=immersion) or not (i. e. for $x \in U_{2}(f)$ there is a neighborhood $U(x)$ of $x$ such that $f \mid U(x)$ is an embedding). For this problem we obtain a following.

Proposition. 1. If $M^{m}, Q^{q}$ are closed $m$ - and $q$-dim. manifolds and if $f: M^{m} \rightarrow Q^{q}(m<q)$ is a map, $f \mid U_{2}(f)$ is an immersion.

Next we consider about almost immersions. Given a map $f: M \rightarrow Q$, we call $f$ almost immersion if $B(f)=S_{2}(f)-U_{2}(f)=$ one point and we call $f$ special almost immersion if $f$ is an almost immersion and if $S_{2}(f \mid l k(a$, $M))=\left\{a_{1}, \cdots, a_{2 n}\right\}$ (finite set of even points) for a unique point $\{a\}=B(f)$ and $S_{3}(f \mid l k(a, M))=\phi$. According to Irwin [1] $f$ is a simple immersion if $S_{3}(f)=\phi=B(f)$. Then we prove

THEOREM 1. Let $M^{m}, Q^{q}$ be $(2 m-q-1)$-connected closed manifolds, $3 m \leqq 2 q-1$ and $q \geqq 6$. Then any $P L$ map $f: M \rightarrow Q$ is homotopic to a special almost immersion $g ; M \rightarrow Q$ with $S_{3}(g)=\phi$.

THEOREM 2. Let $M^{m}$ be $(2 m-q-1)$-connected closed manifold and $Q^{q}$ be $(2 \mathrm{~m}-q)$-connected, $q \leqq m-3$. Then any PL map $f ; M \rightarrow Q$ is homotopic to an almost immersion $g: M \rightarrow Q$ with $S_{3}(g)=\phi$.

In this paper $M, Q$ always mean $m$ - and $q$-dim. closed manifold if otherwise is not stated. $D^{n}, S^{n}$ are always $n$-dim. ball and sphere respectively. For a simplial complex $K$ and a simplex $\Delta^{t}$ in $K$, let

$$
\begin{aligned}
& S t\left(\Delta^{t}, K\right)=\left\{\Delta^{p} \in K \mid \Delta^{p}<\Delta^{q}, \Delta^{q}>\Delta^{t}\right\}, \\
& L k\left(\Delta^{t}, K\right)=\left\{\Delta^{p} \in S t\left(\Delta^{t}, K\right) \mid \Delta^{p} \cap \Delta^{t}=\phi\right\} \text { be }
\end{aligned}
$$

the star and the link of $\Delta^{t}$ in $K$. For spaces or complexes $X$ and $Y, X * Y$ denote a non-singular join of $X$ with $Y$. We call $X * Y$ a linear cone on $Y$ if $X$ is one point. $\partial M$, Int $M$ mean the boundary, the interior of the 
manifold $M$ respectively. Sometime we use the notation $\stackrel{M}{\text { instead of }}$ Int $M$. Both $c l X, \bar{X}$ mean the closure of $X$ in some space.

2. Let $f: M^{m} \rightarrow Q^{q}(m<q)$ be a $P L$ map. It is well known that we can move $f$ into general position by homotopy (i.e. there is a $P L$ map $f_{0}: M \rightarrow Q$ which is homotopic to $f$ and $\operatorname{dim} f_{0}\left(\Delta^{r}\right)=r$ for any $r$-simplex of $M$ and $\operatorname{dim} S_{2}(f) \leqq 2 m-q$.) So unless otherwise is stated we suppose that any $P L$ map $f$ is in general position. Then there is an integer $p$ such that $U_{p+1}(f)=\phi$ since $M$ is compact.

Proof of Propostion. Let $K, J$ be subdivisions of $M, Q$ respectively such that $f: K \rightarrow J$ is simplicial. For $x \in U_{2}(f)$ there is an integer $k \geqq 2$ such that $x \in U_{k}(f)-U_{k+1}(f)$. Since $\operatorname{dim}\left(U_{k}(f)-U_{k+1}(f)\right) \leqq k m-(k-$ 1) $q \equiv r$, there exists a $t$-simplex $\Delta^{t} \in K(t \leqq r)$ such that $x \in \operatorname{Int} \Delta^{t}$. Let $L=$ $L k\left(\Delta^{t}, K\right)$. We first prove that $f \mid L$ is an embedding. Let $\Delta_{i}^{l}, \Delta_{j}^{l} \in L\left(\Delta_{i}^{l} \neq\right.$ $\left.\Delta_{j}^{l}\right)$ with $f\left(\Delta_{i}^{l}\right)=f\left(\Delta_{j}^{l}\right)\left(\operatorname{dim} \Delta_{i}=\operatorname{dim} \Delta_{j}\right.$ because $f$ is non-degenerate). We may assume $l=2 m-q$ since $f$ is in general position. Then $f\left(\Delta_{i}^{l} * \Delta^{t}\right)=f\left(\Delta_{j}^{l} * \Delta^{t}\right)$ and $\operatorname{dim}\left(f\left(\Delta_{i}^{l} * \Delta^{t}\right) \cap f\left(\Delta_{j}^{l} * \Delta^{t}\right)-f\left(\left(\Delta_{i}^{l} \cap \Delta_{j}^{l}\right) * \Delta^{t}\right)=l+t+1>l=2 m-q\right.$. This contradicts to $\operatorname{dim} S_{2}(f) \leqq 2 m-q$. Hence $f \mid L$ is an embedding. Next we show $f\left|\Delta^{t} * L=f\right| S t\left(\Delta^{t}, K\right)$ embedding. $f\left|\Delta^{t} * L=f\right| \cup\left(\Delta_{\Delta_{j} \in L}\left(\Delta^{t} * \Delta_{j}\right)\right.$ and $\Delta^{t} * \Delta_{j}$ is a simplex of $K$ by the definition of $L$. Since $f$ is non-degenerate and simplicial with respect to $K, f \mid \Delta^{t} * \Delta_{j}$ is an embedding. If $P \equiv f\left(\Delta^{t} * \Delta_{i}\right) \cap f\left(\Delta^{t} * \Delta_{j}\right)-$ $f\left(\Delta^{*} *\left(\Delta_{i} \cap \Delta_{j}\right)\right) \neq \phi$ for some $\Delta_{i}, \Delta_{j} \in L, f^{-1}(P) \cap \Delta_{i} \neq \phi$ and $f^{-1}(P) \cap \Delta_{j} \neq \phi$ and $f\left(f^{-1}(P) \cap \Delta_{i}\right)=f\left(f^{-1}(P) \cap \Delta_{j}\right)$ because $f \mid \Delta^{t}$ is an embedding. This contradicts to $f \mid L$ embedding. Hence $f\left|S t\left(\Delta^{t}, K\right)=f\right| S t(x, K)$ is an embedding and so $f \mid U_{2}(f)$ is an immersion.

CoROllary 1. $U_{2}(f) \supset U_{r+1}(f)$ and

$$
S_{2}(f)-U_{2}(f) \supset S_{r+1}(f)-U_{r+1}(f) \text { for any } \quad r \geqq 1 .
$$

Proof. First statement is obvious by definition. To show second statement it is sufficiently to show that $\left(U_{2}(f)-U_{r+1}(f)\right) \cap S_{r+1}(f)=\phi$ for any $r \geqq 1$. Let $P=\left(U_{2}(f)-U_{r+1}(f)\right) \cap S_{r+1}(f)$ and $P \neq \phi$. If $x_{1} \in P, f^{-1} f\left(x_{1}\right)=\left\{x_{1}\right.$, $\left.\cdots, x_{l}\right\}(2 \leqq l \leqq r)$ and for any neighborhood $U\left(x_{1}\right)$ of $x_{1}$, there exists $y_{1} \in$ $U\left(x_{1}\right)$ such that $f^{-1} f\left(y_{1}\right)=\left\{y_{1}, \cdots, y_{p}\right\}, p \geqq r+1(*)$.

On the other hand for the above $x_{i}$ there is a neighborhood $\widetilde{V}\left(x_{i}\right)$ for which $f \mid \widetilde{V}\left(x_{i}\right)$ is an embedding by proposition. We choose a neighdorhood $W\left(f\left(x_{1}\right)\right)$ of $f\left(x_{1}\right)$ in $Q$ so that $W\left(f\left(x_{1}\right)\right) \cap f(M) \subset \bigcup_{i=1}^{l} f\left(\widetilde{V}\left(x_{i}\right)\right)$ and put $V\left(x_{i}\right)=$ $\widetilde{V}\left(x_{i}\right) \cap f^{-1}\left(W\left(f\left(x_{1}\right)\right)\right)$. Then we can show $f^{-1} f\left(y_{1}\right)=\left\{y_{1}, \cdots, y_{q}\right\} \quad(q \leqq l)$ for any $y_{1} \in V\left(x_{1}\right)$. Because $f^{-1} f\left(y_{1}\right) \cap \widetilde{V}\left(x_{1}\right)=y_{1}$ since $f \mid \widetilde{V}\left(x_{1}\right)$ is an embedding. By the same way 


$$
f^{-1} f\left(y_{1}\right) \cap \widetilde{V}\left(x_{i}\right)=\left\{\begin{array}{lll}
y_{i} & \text { if } & f\left(y_{\mathfrak{r}}\right) \in f\left(\widetilde{V}\left(x_{i}\right)\right) \\
\phi & \text { if } & f\left(y_{1}\right) \notin f\left(\widetilde{V}\left(x_{i}\right)\right) .
\end{array}\right.
$$

So $f^{-1} f\left(y_{1}\right) \subset f^{-1}\left(f\left(V\left(x_{1}\right)\right) \cap f(M)\right)=f^{-1}\left(W\left(f\left(x_{1}\right)\right) \cap f(M)\right) \subset \bigcup_{i=1}^{l} f\left(\widetilde{V}\left(x_{i}\right)\right)$.

Hence $f^{-1} f\left(y_{1}\right)=\left\{y_{1}, \cdots, y_{q}\right\}(q \leqq l)$. This contradicts to $\left(^{*}\right)$.

Hence $P=\phi$.

Corollary 2. Let $f: M^{m} \rightarrow Q^{q}(q \geqq m+3)$ be a $P L$ map which is in general position and let $V_{r}(f)=U_{r}(f)-U_{r+1}(f)$. If $V_{r}(f) \neq \phi$ for some $r \geqq 2$, for any $s(2 \leqq s \leqq r) V_{s}(f) \neq \phi$.

Proof. By Proposition $f \mid U_{2}(f)$ is an immersion. For $x \in V_{r}(f)$ let $f^{-1} f\left(x_{1}\right)=\left\{x_{1}, \cdots, x_{r}\right\}$. Then $f \mid S t\left(x_{i}, K\right): S t\left(x_{i}, K\right) \rightarrow S t\left(f\left(x_{1}\right), J\right)$ is a proper locally flat embedding for each $i$ and some triangulations $K, J$ of $M, Q$ respectively. Let $U\left(x_{i}\right)=S t\left(x_{i}, K\right)$ and $U\left(f\left(x_{1}\right)\right)=S t\left(f\left(x_{1}\right), J\right)$. Then by [A-Z, Th. 1.] we can ambient isotope $f\left(U\left(x_{i}\right)\right)$ transversal to $\bigcup_{j \neq i} f\left(U\left(x_{j}\right)\right)$ by small ambient isotopy of $U\left(f\left(x_{1}\right)\right)$. Hence we can consider $U\left(f\left(x_{i}\right)\right) \supset \bigcup_{i}$ $f\left(U\left(x_{i}\right)\right)$ as like as the $m$-dim. planes in $q$-dim. euclidean space $R^{q}$. So let $U\left(f\left(x_{1}\right)\right)=R^{q}$ and $f\left(U\left(x_{i}\right)\right)=E_{i}^{m}(1 \leqq i \leqq r)$. Then there are $(q-1)$-dim, hyperplanes $E_{i, 1}^{q-1}, \cdots, E_{i, q-m}^{q-1}$ such that $E_{i}^{m}=E_{i, 1}^{q-1} \cap \cdots \cap E_{i, q-m}^{q-1}$. For any $k, l(k \neq l)$ $E_{i, k}^{q-1}$ and $E_{i, l}^{q-1}$ are neither parallel nor coincide. Furthermore for any $E_{i, k}^{q-1}$ and $E_{j, l}^{q-1}$ (not $i=j, k=l$ ) they are neither parallel nor coincide. Because for $i=j, k \neq l$, it is obvious. If $i \neq j$ and if $E_{i, k}^{q-1}=E_{j, l}^{q-1}$,

$$
E_{i}^{m} \cap E_{j}^{m}=E_{i, 1}^{q-1} \cap \cdots \cap E_{i, q-m}^{q-1} \cap E_{j, 1}^{q-1} \cap \cdots \cap \hat{E}_{j, l}^{q-1} \cap \cdots \cap E_{j, q-m}^{q-1}
$$

and so $\operatorname{dim}\left(E_{i}^{m} \cap E_{j}^{m}\right)=q-\{(q-m)+(q-m-1)\}=2 m-q+1$. It contradicts $E_{i}^{m}$ and $E_{j}^{m}$ in general position. And if $E_{i, k}^{q-1}$ is parallel to $E_{j, l}^{q-1}, E_{i, k}^{q-1} \cap E_{j, l}^{q-1}$ $=\phi$ and $E_{i}^{m} \cap E_{j}^{m} \subset E_{i, k}^{q-1} \cap E_{j, l}^{q-1}=\phi$. It contradicts to $E_{i}^{m} \cap E_{j}^{m} \neq \phi$. So taking $E_{i_{1}}^{m} \cap \cdots \cap E_{i_{s}}^{m}\left(i_{j} \neq i_{k}\right.$ if $\left.j \neq k, 2 \leqq s \leqq r\right)$,

$$
E_{i_{1}, 1}^{q-1} \cap \cdots \cap E_{i_{1}, q-m}^{q-1} \cap \cdots \cap E_{i_{g}, 1}^{q-1} \cap \cdots \cap E_{i_{s}, q-m}^{q-1} \neq \phi .
$$

Hence $V_{s}(f) \neq \phi$ for $2 \leqq s \leqq r$.

3. Lemma 1. Let $A^{m}, B^{a}$ be balls and let $f: \partial A \rightarrow \partial B$ be a PL map such that $S_{2}(f)=a_{1} \cup a_{2}$. Then there is a special almost immersion $g: A \rightarrow$ $B$ which is an extension of $f$.

Proof. Let $g$ be a linear cone extension of $f$. Then $S_{2}(g)=\left(a * a_{1}\right) \cup$ $\left(a * a_{2}\right)$ where $a$ is the center of $A$ and $S_{2}(g)-\{a\}=U_{2}(g)-U_{3}(g), a \in B(g)$. And it is obvious that $S_{2}(g \mid l k(a, A))$ contains only two points. So $g: A \rightarrow$ $B$ is a sepecial almost immersion which is an extension of $f$. 
LEMMA 2. Let $A^{m}, B^{a}$ be balls and $f: \partial A \rightarrow \partial B$ be a simple immersion whose $S_{2}(f)$ consists of two connected components $C_{1}, C_{2}$ which are $P L$ subspaces. Suppose $3 m \leqq 2 q-1, q \geqq 6$ then there is a special almost immersion $g: A \rightarrow B$ which is an extension of $f$.

Proof. Choose a linear cone $X_{1}^{2 m-q}$ on $C_{1}$ in $\partial A$. Then we may assume $X_{1} \cap C_{2}=\phi$ (if necessary, by putting $X_{1}, C_{2}$ into general position with respect to each other). And remove a second derived neighborhood $N_{1}=N\left(X_{1}, \partial A^{\prime \prime}\right)$ of $X_{1}$ and next put a linear cone $X_{2}^{2 m-q}$ on $C_{2}$ in $\partial A-$ Int $N_{1} \cong D^{m-1}$. Choose a linear cone $Y^{2 m-q+1}$ on $f\left(X_{1} \cup X_{2}\right)$ in $\partial B$. Since by putting $Y$ in general position with respect to $f(\partial A)$

$$
\operatorname{dim}\left(Y \cap f\left(\partial A-\left(X_{1} \cup X_{2}\right)\right)\right) \leqq 2 m-q+1+(m-1)-(q-1) \leqq 0,
$$

let $Y \cap f\left(\partial A-\left(X_{1} \cup X_{2}\right)\right)=\left\{u_{1}, \cdots, u_{r}\right\}$. Then we may assume $u_{i} \notin f\left(S_{2}(f)\right)$. Let $v_{i}=f^{-1}\left(u_{i}\right)$. Joining a point $c_{1} \notin C_{1}$ with $v_{i}$ by simple polygonal arc $\alpha_{i}$ in $\partial A$ so that

$$
\begin{aligned}
& \text { (1) } \stackrel{\circ}{\alpha}_{i} \cap \stackrel{\circ}{\alpha}_{j}=\phi \quad(i \neq j) \\
& \text { (2) } \alpha_{i} \cap X_{1}=c_{1} \\
& \text { (3) } \alpha_{i} \cap X_{2}=\phi \text {, }
\end{aligned}
$$

(it is possible because $q>m+2$ ).

Joining $f\left(c_{1}\right)$ with $u_{i}$ by simple polygonal arc $\beta_{i}$ on $Y$ so that

$$
\begin{array}{ll}
\text { (4) } \stackrel{\circ}{\beta}_{i} \cap \stackrel{\circ}{\beta}_{j}=\phi \quad(i \neq j) \\
\text { (5) } \beta_{i} \cap f(\partial A)=f\left(c_{1}\right) \cup u_{i} .
\end{array}
$$

Since $\operatorname{dim} \partial B=q-1 \geqq 5$, by Embedding Theorem [Z, Chap. 8] there exist $P L$ embeddings $h: D^{2} \rightarrow \partial B(i=1,2, \cdots, r)$ satisfying

$$
\begin{array}{ll}
\text { (6) } & h_{i}\left(\check{D}^{2}\right) \cap h_{j}\left(\dot{L}^{2}\right)=\phi \quad(i \neq j) \\
\text { (7) } & h_{i}\left(\partial D^{2}\right)=f\left(\alpha_{i}\right) \cup \beta_{i} \\
\text { (8) } & h_{i}\left(D^{2}\right) \cap f\left(\partial A-\alpha_{i}\right)=\phi .
\end{array}
$$

let $\widetilde{X}_{1}=X_{1} \cup \bigcup_{i} \alpha_{i}, \widetilde{Y}=Y \cup \bigcup_{i} h_{i}\left(D^{2}\right)$, then $\widetilde{X}_{1} \searrow 0$ in $\partial A, \widetilde{Y} \searrow 0$ in $\partial B$ and $f^{-1}(\widetilde{Y} \cap f(\partial A))^{i}=\widetilde{X}_{1} \cup X_{2}$. So the second derived neighborhood $N=N(\widetilde{Y}$, $\left.\partial B^{\prime \prime}\right), \widetilde{N}_{1}=N\left(\widetilde{X}_{1}, \partial A^{\prime \prime}\right), N_{2}=N\left(X_{2}, \partial A^{\prime \prime}\right)$ of these are $(m-1)$ - and $(q-1)$ balls respectively and $f\left|\tilde{N}_{1}, f\right| N_{2}$ are proper embeddings into $N$. ( $\partial N$ : $\left.f\left(\partial N_{1}\right), f\left(\partial N_{2}\right)\right) \cong\left(S^{q-2}: S^{m-2}, S^{m-2}\right)$ is a link. Let $\widetilde{W}_{1}=N\left(\widetilde{X}_{1}, A^{\prime \prime}\right), W_{2}=N\left(X_{2}\right.$, $\left.A^{\prime \prime}\right), W=N\left(\widetilde{Y}, B^{\prime \prime}\right)$ be second derived neighborhoods of $\widetilde{X}_{1}, X_{2}, \widetilde{Y}$ respectively, then they are all balls. Taking $a_{1} \in \partial \widetilde{W}_{1}-N_{1}, a_{2} \in \partial W_{2}-N_{2}, a \in \partial W$ $-N$, then we may consder $\widetilde{W}_{1} \cong a_{1} * \tilde{N}_{1}, W_{2} \cong a_{2} * N_{2}, W \cong a * N$. So we define a map $\tilde{f}: \partial A \cup \widetilde{W}_{1} \cup W_{2} \rightarrow \partial B \cup W$ by $\tilde{f}=f$ on $\partial A, \tilde{f}\left(a_{1}\right)=\tilde{f}\left(a_{2}\right)=a$ and by 
extending linearly on $\widetilde{W}_{1}, W_{2}$. Then $S_{2}(\tilde{f})=\left(a_{1} * C_{1}\right) \cup\left(a_{2} * C_{2}\right)$ and $\tilde{f}$ is a simple immersion. Let $A_{0}=\overline{A-\left(\widetilde{W}_{1} \cup W_{2}\right)} \cong D^{m}, B_{0}=\overline{B-W}=D^{q}$, then $\tilde{f} \mid \partial A_{0}: \partial A_{0} \rightarrow \partial B_{0}$ is a simple immersion and $S_{2}\left(\tilde{f} \mid \partial A_{0}\right)=a_{1} \cup a_{2}$. So by LEMMA 1 , there is a special almost immersion $\tilde{g}: A_{0} \rightarrow B_{0}$ such that $\tilde{g} \mid \partial A$ $=\tilde{\boldsymbol{f}} \mid \partial A_{0}$.

We define a map $g: A \rightarrow B$ by

$$
g=\left\{\begin{array}{lll}
\tilde{f} & \text { on } & \partial A \cup \widetilde{W}_{1} \cup W_{2} \\
\tilde{g} & \text { on } & A_{0}
\end{array}\right.
$$

It is clear that $g$ is a special almost immersion.

REMARK. 1. Even if $S_{2}(f)$ has as connected component more than 2, it is clear that we may deal separately with each pair of connected components which is identified under $f$. So LEMMA 1 and LEMMA 2 may be carried out well clear of even components more than 2 .

REMARK. 2. Let $A^{m}, B^{q}$ balls and $f: \partial A \rightarrow \partial B$ be an immersion, then there is an almost immersion $g: A \rightarrow B$ using cone extension such that $g \mid \partial A=f$.

Proof of Theorem 1. Since $f$ is in general position, $\operatorname{dim} S_{2}(f) \leqq$ $2 m-q$ and $S_{3}(f)=\phi$. And the branch locus $B(f)=S_{2}(f)-U_{2}(f)$ is contained in the $(2 m-q-1)$-skeletion of some triangulation of $M$. Using Engulfing Theorem ([Z. Chap. 7]) there is a collapsible subpolyhedron $X^{2 m-q}$ in $M$ which contains $B(f)$. Since $\operatorname{dim}\left(X \cap\left(S_{2}(f)-B(f)\right)\right) \leqq 3 m-2 q<0$, $f \mid X$ is an embedding and so $f(X) \searrow 0$ in $Q$. Now put $A^{m}=N\left(X, M^{\prime \prime}\right), B^{q}$ $=N\left(f(X), Q^{\prime \prime}\right)$ then $A, B$ are $m$-and $q$-balls and by the map $f: M \rightarrow Q$, $f(M-A) \subset Q-B, f(\partial A) \subset \partial B$ and $f(\operatorname{Int} A) \subset \operatorname{Int} B$. Furthermore $B(f) \subset \operatorname{Int} A$. Since $f \mid M-$ Int $A$ is a simple immersion, by Lemma 2 and Remark 1 there is a special almost immersion

$$
\tilde{g}: A \rightarrow B \text { such that } \tilde{g} \mid \partial A=f .
$$

So we define $f: M \rightarrow Q$ by

$$
g=\left\{\begin{array}{lll}
f & \text { on } & M-\operatorname{Int} A \\
\tilde{g} & \text { on } & A
\end{array}\right.
$$

Then $g$ is a special almost immersion which is homotopic to $f$.

Remark. Let $f: M^{m} \rightarrow Q^{a}$ be a $P L$ map with $S_{3}(f)=\phi$. Then a connected component $\widetilde{C}$ of $f\left(U_{2}(f)\right)$ is a connected manifolds. We denote $f^{-1}(\widetilde{C})=C_{1} \cup C_{2}$ where $C_{i}(i=1,2)$ are subset of $f^{-1}(\widetilde{C})$ satisfping the following conditions, 
1. ${ }^{v} c_{1} \in C_{1} \quad{ }^{\exists} c_{2} \in C_{2} \ni f\left(c_{1}\right)=f\left(c_{2}\right)$

2. ${ }^{v} d_{2} \in C_{2} \quad{ }^{\sharp} d_{1} \in C_{1} \ni f\left(d_{1}\right)=f\left(d_{2}\right)$

3. $C_{i}(i=1,2)$ are connected and $C_{1} \cap C_{2}=\phi$.

Then we may consider following cases.

CASE 1. If $C_{1}$ is a closed subset in $f^{-1}(\widetilde{C})$ or if $C_{1}$ is an open subset in $f^{-1}(\widetilde{C}), f^{-1}(\widetilde{C})$ is not connected.

CASE 2. If $C_{1}$ is neither closed nor open in $f^{-1}(\widetilde{C}), f^{-1}(\widetilde{C})$ is connected. Since $f$ is a $P L$ map, $\partial C_{i} \equiv C l\left(C_{i}\right)-\operatorname{Int}\left(C_{i}\right)$ is contained in the $(2 m-q-1)$-skeleton of some triangulation of $M$. So if CASE 2 happens to Theorem 1 , we take $X^{2 m-q}$ so that it contains not only $B(f)$ but also $\partial C_{i}$. Then LEMMA 2 is available.

Proof of Theorem 2. Since $f: M \rightarrow Q$ is simplicial and in general position with respect to some triangulations of $M$ and $Q, \operatorname{dim} B(f) \leqq$ $2 m-q-1$ and by Engulfing Theorem ([Z Chap. 7]) there is a collapsible polyhedron $X_{0}$ in $M$ which contains $B(f)$. And there is a collapsible polyhedron $Y_{0}^{2 m-q+1}$ in $Q$ which contains $f\left(X_{0}\right)$. Put $W_{0}=\left(f^{-1}\left(Y_{0}\right)-X_{0}\right)$ then

$$
\operatorname{dim} W_{0} \leqq 2 m-q-2 \text { and so } \operatorname{dim} W_{0} \leqq \operatorname{dim} B(f) .
$$

We prove the theorem by induction as follows. Inductive hypothesis $\phi(i)$ : There exist collapsible subspaces $X_{i}$ and $Y_{i}$ such that $B(f) \subset X_{i} \subset M, f\left(X_{i}\right)$ $\subset Y_{i} \subset Q$ and $W_{i}=C l\left(f^{-1}\left(Y_{i}\right)-X_{i}\right)$ has dimension $\leqq 2 m-q-i-2$. The case $i=0$ has been proved above. We assume $\phi(j-1), j \geqq 1$ and prove $\phi(j)$. $\operatorname{dim} W_{j-1} \leqq 2 m-q-j-1$. By Engulfing Therem there is a subspace $\widetilde{X}_{j}^{2 m-q-j}$ in $M$ such that $W_{j-1} \subset \widetilde{X}_{j}^{2 m-q-j}$ and $X_{j}=X_{j-1} \cup \widetilde{X}_{j} \searrow 0$. And there is a subspace $\widetilde{Y}_{j}^{2 m-q-j+1}$ in $Q$ such that $f\left(\widetilde{X}_{j}\right) \subset \widetilde{Y}_{j}$ and $Y_{j}=Y_{j-1} \cup \widetilde{Y}_{j} \searrow 0$. Then $f\left(X_{j}\right) \subset Y_{j}$ and we put $Y_{j}$ rel. $f\left(\widetilde{X}_{j}\right) \cup Y_{j-1}$, into general pasition with respect to $f(M)$. Now $\left(Y_{j} \cap f(M)\right)-f\left(X_{j}\right)=\left(\widetilde{Y}_{j} \cap f(M)\right)-f\left(X_{j}\right)$ and $\operatorname{dim} W_{j}$ $=\operatorname{dim}\left(\left(Y_{j} \cap f(M)\right)-f\left(X_{j}\right)\right) \leqq 2 m-q-j+1+m-q \leqq 2 m-q-j-2$.

$\phi(2 m-q-1)$ tell us that $W_{2 m-q-1}=\phi$ and so $B(f) \subset X_{2 m-q-1} \searrow 0, f\left(X_{2 m-q-1}\right) \subset$ $Y_{2 m-q-1} \searrow 0$ and $f^{-1}\left(Y_{2 m-q-1}\right)=X_{2 m-q-1}$. We put $X=X_{2 m-q-1}, Y=Y_{2 m-q-1}$ and let $A=N\left(X, M^{\prime \prime}\right), B=N\left(Y, Q^{\prime \prime}\right)$ ( $m$-and $q$-balls respectively). Then $f(M-$ $A) \subset Q-B, f(\partial A) \subset \partial B, f(A) \subset B$ and $B(f) \subset \operatorname{Int} A$. Since $f \mid \overline{M-A}$ is an immersion, by REMARK 2 we can extend $f \mid \partial A$ to an almost immersion $\tilde{f}: A \rightarrow B$ and we obtain a required almost immersion

$$
\begin{aligned}
& g: M \rightarrow Q \text { by defining } \\
& g=\left\{\begin{array}{lll}
f & \text { on } & M-A \\
\tilde{f} & \text { on } & A .
\end{array}\right.
\end{aligned}
$$


In particular taking $X_{0} \supset B(f) \cup S_{3}(f)$ because $3 m-2 q<2 m-q-1, g$ is an almost immersion with $S_{3}(g)=\phi$.

EXAmple. It is well known that $n$-dim. complex projective space $P^{n}(C)$ is immersible in $R^{4 n-1}$ but not $R^{4 n-2}$ for $n=2^{r}$. On the other hand Theorem 1 and Theoem 2 tell us that $P^{n}(C)$ is special almost immersible in $R^{4 n-2}$ for $n=2^{r}, r \geqq 2$.

\section{References}

[A-Z] Armstrong, M. A. and Zeeman, E. C.: Transversality for piecewise linear manifolds. Topology Vol. 6 (1967), pp. 433-466.

[I] IRwin, M. C.: Polyhedral immersions, Proc. Camb. Phil. Soc. (1966), Vol. 62. pp. $45-50$.

[ z ] Zeeman, E. C.: Seminar on combinatorial topology (mimeographed note), Institute des Hautes Etudes Scientifiques, Paris (1963).

Department of Mathematics Hokkaido University 